\title{
MMNS Posters
}

1. An approach for Implicit and Explicit Activation of Service Level Agreements

Dimitrios Kagklis, Cristos Tsakiris, Nicolas Liampotis, Efstathios Sykas

Telecommunications Lab.Dept of Electrical \& Computer Engineering National

Technical University of Athens

Zografou Campus, Heroon Polytechniou 9, 15773 Athens, Greece

Email: kaglis,chrtsak,nliam,sykas@telecom.ntua.gr

2. A Multi-Domain Service Session Accounting \& Charging Architecture

M. van Le ${ }^{1}$, B.J.F van Beijnum ${ }^{1}$, G.B. Huitema ${ }^{2}$

1 Department of Computer Science, University of Twente, Netherlands

2 Department of Management and Organization University of Groningen,

Netherlands

Email: le,beijnum@cs.utwente.nl; g.b.huitema@bdk.rug.nl

3. New formal approach for QoS management in Distributed Multimedia Applications

Sophie Laplace, Marc Dalmau, Philippe Roose

Laboratoire d'Informatique de l'Université de Pau et des Pays de l'Adour

Avenue de l'Université, B.P. 1155, 64013 Pau Cedex, France

Email: laplace, dalmau, roose@iutbayonne.univ-pau.fr

4. Management of Security in TCP/IP Hosts Using Dedicated Monitoring Applications

Rui Cardoso, Mario Freire

Networks and Multimedia Group, Institute of Telecommunications at Coimbra,

Department of Informatics, University of Beira Interior,

Rua Marques D'Avila e Bolama,

P-6200-001 Covilhã, Portugal

Email: rcardoso, mario@di.ubi.pt

5. Mobility/Roaming Parameters and constraints in a Service Level Agreement

Rima Tfaily Souayed ${ }^{1}$, Dominique Gaiiti ${ }^{1,2}$ and Guy Pujolle ${ }^{2}$

1.Université de technologies de Troyes (UTT) - LM2S 12 rue Marie Curie, BP 2060

- 10010 Troyes Cedex, France

2. Université Pierre et Marie Curie, 8 rue du Capitaine Scott, 75015 Paris, France

Email: rima.tfaily, dominique.gaiti@utt.fr, guy.pujolle@lip6.fr 
6. Event Management Middleware Services for Proactive Management

Dugki Min ${ }^{1}$, Eunmi Choi ${ }^{2}$

1 School of Computer Science and Engineering, Konkuk. University, Hwayang-dong, Kwangjin-gu, Seoul, 133-701, Korea

2 School of Computer Science and Electronic Engineering,

Handong Global University,

Heunghae-eub, Puk-ku, Pohang, Kyungbuk 791-940, Korea

Email: dkmin@konkuk.ac.kr, emchoi@handong.edu

\section{A Vendor and Consumers Dream: Web based Service Provision}

Stephen Dawson, Sakir Sezer

School of Electrical and Electronic Engineering

Queen's University Belfast

Ashby Building, Stranmillis Road

Belfast, BT9 5AH, N. Ireland, U.K.

Email: Stephen.Dawson@ee.qub.ac.uk

8. Dynamic Reconfiguration of IP Domain Middleware Stacks to Support Multicast Multimedia Distribution in a Heterogeneous Environment

Kevin Curran, Gerard Parr

Telecommunications \& Distributed Systems Research Group

Northern Ireland Knowledge Engineering Laboratory

University of Ulster, Magee Campus, Northern Ireland, BT48 7JL, UK

Email: kj.curran@ulst.ac.uk

9. Proactive Network Diagnosis Model Using Fuzzy Logic in Mobile Agents and Remote Objects

Arnoldo Nunes da Silva ${ }^{1}$, Carlos André Guimarães Ferraz ${ }^{2}$,

Geber Lisboa Ramalho ${ }^{2}$, José Neuman de Souza ${ }^{3}$

1 National Network of Research - Federal University of Ceará Campus do PICI, Bl. 901 - ZIP: 60455-760 - Fortaleza - Ce - Brazil

2 Center of Informatics - Federal University of Pernambuco P.O.BOX: 7851 ZIP: 50732-970 - Recife - Pe - Brazil

3 Department of Computer Science - Federal University of Ceará Campus do PICI, Bl. 910 ZIP: 60455-760 - Fortaleza - Ce - Brasil

Email : arnoldo@ufc.br, cagf,glr@cin.ufpe.br, neuman@ufc.br 